\title{
RETIFICADORES DE ALTA QUALIDADE COM COMUTAÇÃO EM ALTA OU EM BAIXA FREQÜÊNCIA - UM ESTUDO COMPARATIVO
}

\author{
José Antenor Pomilio \\ Faculdade de Engenharia Elétrica e de Computação \\ Universidade Estadual de Campinas \\ C. P. 6101 13081-970 Campinas - Brasil \\ e-mail: antenor@dsce.fee.unicamp.br \\ Giorgio Spiazzi, Simone Buso \\ Departamento de Eletrônica e Informática \\ Universidade de Pádua \\ Via Gradenigo 6A - 35131 Padova - Itália \\ e-mail: giorgio@dei.unipd.it - simone@.dei.unipd.it
}

\begin{abstract}
Resumo - Retificadores com alto fator de potência, em conformidade com normas internacionais têm sido largamente estudados. São bastante conhecidas as soluções que utilizam comutação em alta frequiência, enquanto são mais recentes as topologias que utilizam comutação em baixa freqüiência. Estas últimas representam uma alternativa para aplicações de grande volume de produção nas quais não é necessária uma regulação precisa da tensão de saída, sendo possível atender às limitações da norma com valores de indutância inferiores àqueles usados em soluções passivas. Além disso, devido à operação em baixa frequiência, as perdas de comutação se tornam desprezíveis e os valores de di/dt e dv/dt são inferiores aos dos circuitos comutados em alta freqüência, minimizando a emissão de interferência eletromagnética. Este artigo revisa os princípios de funcionamento de alguns retificadores deste tipo. São feitas comparações com a solução de alta freqüência em termos de complexidade circuital, volume dos indutores e desempenho, permitindo estabelecer critérios de escolha dentre as topologias.
\end{abstract}

\begin{abstract}
High power factor rectifiers, complying with international standards have been deeply studied. Two main approaches are usually used: high frequency control of the input current and line-frequency commutated rectifiers. These last represent an alternative for large volume applications that do not need a precise output voltage regulation. They provide compliance with the standard using a smaller inductor as compared to a conventional rectifier with passive filter. Moreover, being the switch turned on and off only twice per line period, the associated losses are very small and the di/dt and dv/dt are lower compared to high-frequency commutated rectifiers, thus reducing the high-frequency noise emission and EMI filter requirements. This paper reviews the operating principles of some line-frequency commutated rectifier topologies. A comparison among low and high frequency high power factor rectifiers, in terms of circuit complexity, overall reactive component size and performance, is made, thus allowing selection of the most convenient topology for a given application.
\end{abstract}

\section{INTRODUÇÃO}

Retificadores de alta qualidade (em inglês "Power Factor Correctors - PFCs") têm substituído retificadores com filtro capacitivo devido aos limites para as componentes harmônicas impostos por normas internacionais como a IEC-61000-3-2 [1]. Os PFCs com comutação em alta freqüência levam a um fator de potência praticamente unitário, além de permitirem regular a tensão de saída, às custas de um aumento no custo e no volume total do retificador. O PFC baseado no conversor boost operando no modo de condução contínuo (MCC) com comutação em alta freqüência ( $\mathrm{AF}$ - boost), é a estrutura apontada na literatura como das mais indicadas para a implementação de um retificador de alta qualidade, devido à inerente baixa ondulação da corrente de entrada, à excelente forma de onda e à facilidade de comando do transistor.

Não obstante, algumas aplicações de produção maciça, como eletrodomésticos de maior potência, da chamada "linha branca" (aparelhos de ar condicionado, máquinas de lavar roupa, etc.) ainda utilizam topologias convencionais devido ao menor custo e maior confiabilidade, com filtros passivos para se conformarem à norma, mesmo que isto signifique um significativo aumento no volume dos elementos reativos na medida em que aumenta a potência [2].

Recentemente [3-7] foram propostas topologias que representam uma solução de compromisso entre os retificadores com comutação em alta freqüência (que normalmente comutam em dezenas de $\mathrm{kHz}$ ) e os com filtros passivos. Tais circuitos fazem uso de um interruptor comutado no dobro da freqüência da rede, de modo que praticamente são eliminadas as perdas por comutação. O atendimento às especificações da norma é obtido com importante redução no valor dos elementos reativos, especialmente indutâncias, quando comparado com a solução ativa. Além disso, os limitados valores de di/dt e dv/dt permitem a minimização de emissões de alta freqüência, possivelmente eliminando a necessidade de filtros de linha.

Circuitos deste tipo têm sido utilizados industrialmente, como se verifica em [8] para o caso de aparelhos de ar condicionado.

Algumas alternativas para conversores trifásicos também têm sido estudadas [9-11] mas fogem do escopo deste artigo pois normalmente não ocorrem em aplicações domésticas. 
Neste artigo são revistas e analisadas quatro destas topologias. Uma comparação com o AF - boost é realizada, observando aspectos como: distorção na corrente de entrada, fator de potência, regulação da tensão de saída, complexidade do circuito, esforços de tensão e de corrente nos interruptores, tamanho dos elementos reativos, etc. São estudados casos na faixa de potência entre $600 \mathrm{~W}$ e $1,2 \mathrm{~kW}$.

\section{RETIFICADORES COM COMUTAÇÃO EM BAIXA FREQÜÊNCIA}

Além do AF - boost, as demais topologias de retificadores que serão consideradas neste artigo estão mostradas na figura 1. Cada uma possui um indutor, L e um capacitor, C, ambos dimensionados para operação em baixa frequiência. Além disso, as topologias T1 e T2 são comutadas em baixa freqüência e utilizam um circuito auxiliar que emprega um transistor, S e um diodo, D.

A topologia T2 também emprega indutor e capacitor auxiliares, $\mathrm{L}_{\mathrm{a}}$ e $\mathrm{C}_{\mathrm{a}}$, respectivamente. Exceto pelo diodo $\mathrm{D}$, todos os demais componentes são dimensionados para uma pequena fração da potência de saída.

Estes conversores permitem atender aos limites impostos pelas normas de distorção harmônica da corrente, fazendo uso de componentes passivos ( $\mathrm{L}$ e C) de valores inferiores aos utilizados em circuitos que empregam apenas filtragem passiva para o mesmo fim. Dado que a corrente de entrada é descontínua, o posicionamento da indutância pode ser feito tanto do lado CA quanto do lado CC do retificador.

Os princípios de funcionamento são brevemente descritos a seguir.

Topologia $T 1$ - boost com comutação em baixa freqüência $(B F-b o o s t)$ : Este circuito é topologicamente idêntico ao conversor boost com comutação em alta freqüência, no entanto o comando do transistor se faz no dobro da frequiência da rede, ou seja, apenas uma comutação em cada semiciclo. Quando o transistor entra em condução, a tensão retificada da rede, $u_{g}$, é aplicada à indutância e se tem corrente absorvida da rede com antecedência em relação ao que ocorreria sem a ação deste interruptor. Quando o transistor é desligado, ocorre uma ressonância entre L e C, através do diodo, até que a corrente se anule, assim permanecendo até o próximo semiciclo. A forma de onda típica está mostrada na figura 2.a).

Topologia $T 2$ - (BF - boost modificado): Este conversor opera de forma diversa do anterior. O interruptor $S$ é acionado nas proximidades do cruzamento da tensão da rede com o zero. Isto provoca uma descarga (parcial) do capacitor $\mathrm{C}_{\mathrm{a}}$ através do indutor La. Quando o transistor é desligado o processo de descarga prossegue através de $D_{a}$ até que a corrente pelo indutor auxiliar vá a zero. Ao final deste intervalo o capacitor está carregado com uma fração da tensão de saída, levando a uma condução antecipada dos diodos da ponte retificadora. A corrente de entrada varia de maneira ressonante, carregando $\mathrm{C}_{\mathrm{a}}$, até que o diodo $\mathrm{D}$ entre em condução. Então se tem a ressonância entre $\mathrm{L}$ e $\left(\mathrm{C}+\mathrm{C}_{\mathrm{a}}\right)$, que se mantém até que a corrente se anule. Formas de onda típicas estão mostradas na figura 2.b). a)

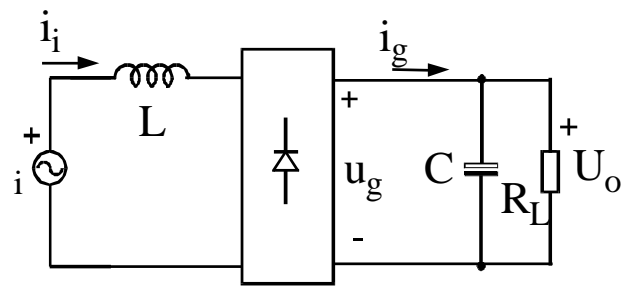

b)

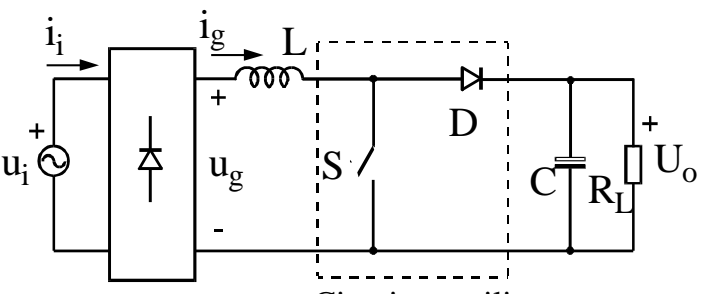

Circuito auxiliar

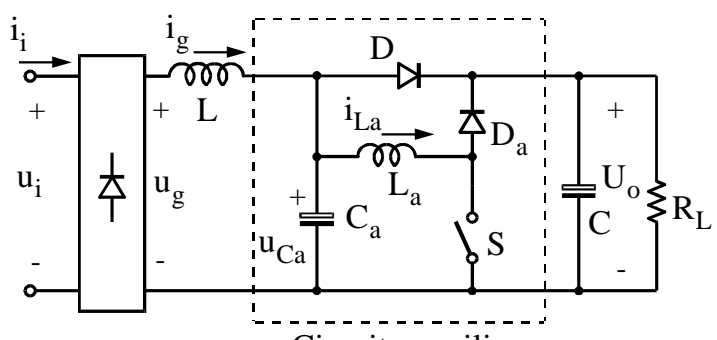

c)

Circuito auxiliar

Fig. 1 -Topologias de retificados de alto fator de potência: a) Filtro passivo; b) T1: BF- boost; c) T2: BF - boost modificado.

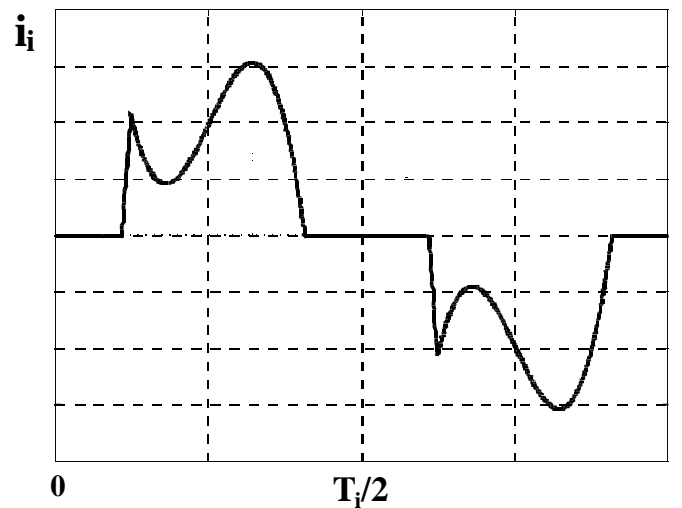

a)

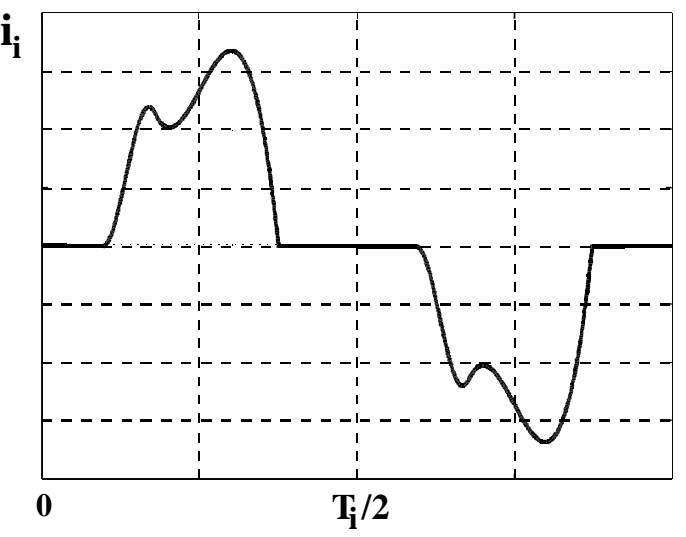

Fig. 2 - Formas de onda típicas da corrente de entrada dos retificadores com comutação em baixa frequiência: a) T1; b) T2. 


\section{COMPARAÇÕES ENTRE AS TOPOLOGIAS}

As tabelas I e II mostram dados das quatro topologias selecionadas para a análise comparativa, incluindo aquela com filtro passivo (LC com célula única). Todos os valores referem-se a uma tensão de entrada de $230 \mathrm{~V}$, como prescreve a norma.

O valor da indutância é o mínimo que permite a obediência aos limites da norma (classe A), tendo sido obtido por método numérico, seguindo procedimentos indicados em [4-7]. Para o conversor boost de alta freqüência, operando no MCC, o valor da indutância foi calculado considerando os seguintes valores: ondulação da corrente de entrada (pico-apico) de $2,6 \mathrm{~A}$, a $70 \mathrm{kHz}$, com tensão de saída de $380 \mathrm{~V}$.

\section{A. Conformidade às normas de distorção harmônica da corrente}

Para o conversor com comutação em alta freqüência, supondo operação no modo de condução contínuo, com controle pela corrente média, a corrente da rede reproduz a forma de onda da tensão de entrada, o que garante o atendimento das restrições da norma. No modo de condução descontínuo, depois da filtragem da alta freqüência, tem-se uma distorção harmônica mais elevada [12], como mostra a figura 3 , mas muito aquém dos limites da norma.

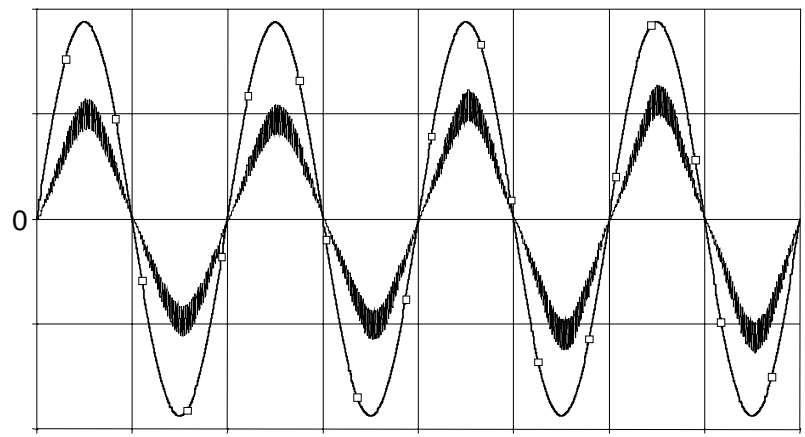

Fig. 3 - Forma de onda típica de retificador "boost" no modo de condução descontínuo: tensão da rede e corrente filtrada.

A emenda 14, introduziu alterações na norma IEC 610003-2 [13], determinando que apenas aparelhos de TV e computadores e monitores de uso pessoal, com potência até 600 W são incluídos na classe D. Nesta classe os limites estabelecidos para cada harmônica são uma percentagem da componente fundamental.

Foi abolida a definição do envelope dentro do qual a corrente deveria se conformar, desta forma, não existe mais a possibilidade de se alterar ligeiramente a forma de onda de modo a passar-se da classe D para a classe A.

Para os outros aparelhos (exceto os de iluminação classe C, as ferramentas portáteis - classe B e os da classe D), o equipamento é considerado em classe A, existindo valores absolutos a serem respeitados, independentemente da potência. Por exemplo, a terceira harmônica pode ter um valor de pico de até $3,25 \mathrm{~A}$.

Utilizando os valores indicados na Tabela I, foram verificados os espectros das correntes de entrada de um retificador com filtro passivo e das topologias $\mathrm{T} 1$ e $\mathrm{T} 2$, garantindo-se a mesma potência de saída (para 1200 W). Os resultados estão mostrados na figura 4.

Pode-se verificar que para o circuito com filtro passivo o limite é dado pela componente de mais baixa ordem $\left(3^{\mathrm{a}}\right.$ harmônica). A forma suave da onda limita fortemente as componentes superiores.

$\mathrm{Na}$ topologia $\mathrm{T} 1$ verifica-se que a redução nas componentes de baixa ordem vem acompanhada de uma elevação daquelas de ordem superior. Neste caso o limite foi dado pela $17^{\mathrm{a}}$ harmônica.

$\mathrm{Na}$ topologia $\mathrm{T} 2$, por também apresentar um perfil mais suave de variação da corrente em relação a T1, este efeito de aumento nas componentes de ordem elevada não se observa, e o limite de potência é dado pelas $5^{\mathrm{a}}$ e $7^{\mathrm{a}}$ harmônicas, que estão associadas à ondulação da forma de onda mostrada na figura 2 .

Observe-se que, de acordo com a Tabela I, a conformidade com a norma é obtida com indutância cada vez menor quando se passa do circuito passivo para as topologias T1 e T2.

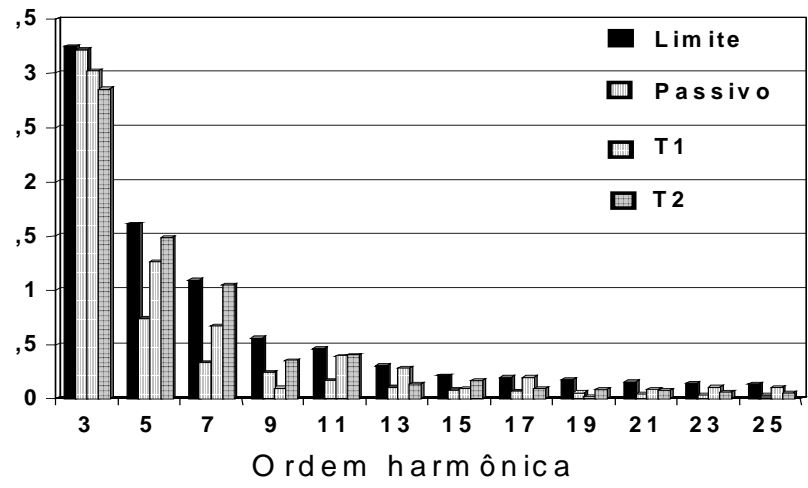

Fig. 4 - Comparação entre espectros da corrente de entrada de retificadores com filtro passivo, topologias $\mathrm{T} 1 \mathrm{e} \mathrm{T} 2 \mathrm{e}$ os limites da norma (valores de pico em $[\mathrm{A}]$ ). $\mathrm{P}_{\mathrm{o}}=1200 \mathrm{~W}$.

\section{B. Regulação da tensão de saída}

Para o AF - boost é possível se obter uma excelente regulação da tensão de saída, tanto para variações na carga quanto para variações na entrada.

A solução passiva não oferece nenhum tipo de regulação e, além disso, devido à queda de tensão sobre a indutância, a tensão CC é significantemente menor do que o valor de pico da tensão de entrada.

O efeito boost (elevação da tensão de saída em relação à de entrada) está presente também nos conversores com comutação em baixa freqüência. A energia armazenada durante o intervalo de condução do interruptor é transferida subseqüentemente à saída, elevando a tensão CC.

Esta elevação, por depender da energia acumulada na indutância, está diretamente ligada à duração do intervalo de condução do transistor, o qual é limitado pelo máximo esforço de corrente admitido para este dispositivo, como será discutido no próximo item. Desta maneira, é possível se obter regulação da tensão de saída, numa faixa relativamente ampla de variação da carga mas numa faixa limitada de variação da tensão da entrada.

Uma vez que o critério de dimensionamento da indutância é o de minimizar seu valor, mantida a conformidade com a norma, o tempo de condução do 
interruptor é relativamente curto, uma vez que tempos maiores exigiriam indutâncias mais elevadas com a finalidade de limitar a corrente que circularia pelo interruptor. Sendo pequeno este intervalo de tempo, sua redução pode compensar um aumento na tensão de entrada apenas numa pequena faixa. Não é possível fazer compensação de sub-tensão, pois isso implicaria no aumento do tempo de condução, com o conseqüente aumento no esforço de condução de corrente.

Em termos da variação da carga, na medida em que a corrente da carga se reduz, o tempo de condução do transistor deve ser reduzido de maneira a manter constante a tensão de saída. Para um dado valor mínimo de corrente consumida, o intervalo $t_{\text {on }}$ se anula e o retificador passa a se comportar como um conversor com filtro passivo. Abaixo deste valor de corrente da carga não é possível regular a tensão de saída, a qual tende a se elevar, chegando ao valor de pico da tensão de entrada.

Quanto maior o valor da indutância do circuito, maior a queda de tensão sobre esta. Assim, na topologia T1 verificase uma significativa diminuição da tensão CC, enquanto em T2 consegue-se aproximar do valor da tensão de pico da entrada em toda faixa de potência estudada.

\section{Esforços de tensão de corrente}

Para os circuitos com comutação em baixa freqüência, o esforço de corrente do interruptor depende tanto do valor da indutância quanto do tempo de condução do transistor. Quanto maior for este intervalo de tempo maior a faixa de regulação da tensão de saída. Mas isto implica em um maior valor de pico da corrente pelo indutor ou em uma maior indutância, o que traz implicações no aumento do volume do indutor.

A topologia T2 apresenta um maior esforço de corrente, uma vez que utiliza indutância menor através da qual se faz a descarga do capacitor auxiliar.

A figura 5 mostra formas de onda de tensão e de corrente no circuito auxiliar. Além do transistor e do diodo, também o capacitor fica submetido a um importante esforço de corrente, indicando a necessidade de uso de um componente com baixa resistência série equivalente a fim de limitar as perdas neste componente.

O esforço de corrente para o AF - boost, no modo de condução contínuo, é igual ao valor de pico da corrente pelo indutor. O esforço de tensão ao qual estão submetidos os interruptores é igual à tensão de saída para todos os três conversores, mas normalmente será maior para o caso de alta frequiência devido à maior tensão de saída obtida.

\section{Fator de Potência}

Considerando as formas de onda mostradas na figura 2, é possível determinar as respectivas distorções harmônicas totais (DTH), fatores de deslocamento da componente fundamental $\left(\cos \phi_{1}\right)$ e os conseqüentes fatores de potência (FP).

Consultando a Tabela I, verifica-se que os resultados para o AF - boost são os melhores, enquanto a topologia com filtro passivo apresenta o pior desempenho em termos de fator de deslocamento e fator de potência. Devido à forma de onda suave, sua DHT é inferior às das topologias T1 e T2.

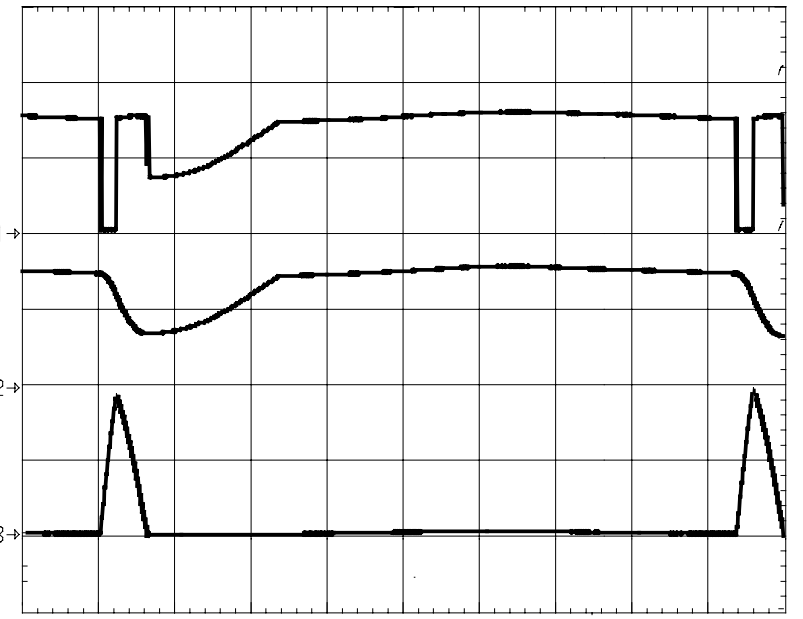

Fig. 5 - Formas de onda no circuito auxiliar: $\{1\}$ : Tensão no transistor (200 V/div.)

$\{2\}$ : Tensão no capacitor auxiliar (200 V/div.)

$\{3\}$ : Corrente no indutor auxiliar (20 A/div.) Horiz.: $1 \mathrm{~ms} / \mathrm{div}$.

Quanto aos retificadores com comutação em baixa freqüência, os resultados para T2 são melhores do que para T1. Isto se explica considerando que a corrente de entrada para a topologia T2 apresenta-se com menores valores de pico e eficaz, o que implica numa redução da distorção e um aumento do FP.

\section{E. Tamanho do indutor}

Uma estimativa do tamanho dos indutores pode ser obtida por meio do produto de áreas $\mathrm{A}_{\mathrm{e}} \mathrm{A}_{\mathrm{w}}$ (sessão transversal do núcleo x área da janela ocupada pelo enrolamento), bastante utilizado nos procedimentos de dimensionamento deste tipo de elemento.

A tabela I mostra os valores deste produto considerando os parâmetros e características dados a seguir. Para os conversores com operação em baixa frequiência considera-se um núcleo de Ferro - Silício, uma densidade de campo magnético $\mathrm{B}_{\max }=1,5 \mathrm{~T}$, uma densidade de corrente $\mathrm{J}=5$ $\mathrm{A} / \mathrm{mm}^{2}$, e coeficiente de ocupação da janela pelo enrolamento $\mathrm{k}_{\mathrm{R}}=0,4$.

Para o AF - boost, considera-se o emprego de ferrite (Micrometals) com densidade de campo magnético $\mathrm{B}_{\max }=$ $0,15 \mathrm{~T}$, no caso de potência de saída de $600 \mathrm{~W}$. Um núcleo de pó de ferro, tipo kool- $\mu$ (Magnetics) com $B_{\max }=0,6 \mathrm{~T}$ foi considerado para potências mais elevadas (900 e 1200 W). A elevação de temperatura admitida é de $40{ }^{\circ} \mathrm{C}$.

$\mathrm{Na}$ tabela II têm-se estimativas dos volumes externos do indutor e apenas do núcleo, com base em valores típicos de produtos comerciais.

Como esperado, o conversor com comutação em alta freqüência apresenta os menores valores de volume do indutor. A diferença se torna mais significativa à medida que aumenta a potência.

Dentre as soluções de baixa freqüência, T2 é a que apresenta menores valores, mesmo adicionando-se no cômputo o volume do indutor auxiliar. 
TABELA I. COMPARAÇÃO ENTRE RETIFICADORES PARA DIFERENTES POTÊNCIAS: ESFORÇOS DE TENSÃO E DE CORRENTE E FATOR DE POTÊNCIA

\begin{tabular}{|c|c|c|c|c|c|c|c|c|c|c|c|c|c|c|c|}
\hline $\begin{array}{c}\mathrm{P}_{\mathrm{o}} \\
{[\mathrm{W}]}\end{array}$ & $\begin{array}{c}\mathrm{U}_{\mathrm{o}} \\
{[\mathrm{V}]}\end{array}$ & $\begin{array}{c}\mathrm{U}_{\mathrm{o}} / \mathrm{U}_{\mathrm{Spk}} \\
{[\mathrm{V}]}\end{array}$ & $\begin{array}{c}\mathrm{L} \\
{[\mathrm{mH}]}\end{array}$ & $\begin{array}{c}\mathrm{L}_{\mathrm{a}} \\
{[\mathrm{mH}]}\end{array}$ & $\begin{array}{c}\mathrm{I}_{\text {gpeak }} \\
{[\mathrm{A}]}\end{array}$ & $\begin{array}{l}\mathrm{I}_{\text {grms }} \\
\text { [A] }\end{array}$ & $\begin{array}{l}\mathrm{I}_{\text {gavg }} \\
{[\mathrm{A}]}\end{array}$ & $\begin{array}{c}\mathrm{I}_{\text {Speak }} \\
{[\mathrm{A}]}\end{array}$ & $\begin{array}{c}\mathrm{I}_{\text {Lapeak }} \\
{[\mathrm{A}]}\end{array}$ & $\begin{array}{c}\mathrm{I}_{\text {Larms }} \\
{[\mathrm{A}]}\end{array}$ & DHT & $\cos \left(\phi_{1}\right)$ & FP & $\begin{array}{l}\mathrm{A}_{\mathrm{e}} \mathrm{A}_{\mathrm{w}} \\
{\left[\mathrm{cm}^{4}\right]}\end{array}$ & $\begin{array}{l}\mathrm{A}_{\mathrm{e}} \mathrm{A}_{\mathrm{w}} \\
{\left[\mathrm{cm}^{4}\right]}\end{array}$ \\
\hline $600-P$ & 294,8 & 0,90 & 6,5 & & 8,62 & 3,68 & 2,04 & & & & 0,87 & 0,938 & 0,708 & 6,87 & \\
\hline $600-T_{1}$ & 303,4 & 0,93 & 4,5 & & 8,84 & 3,63 & 1,98 & 1,33 & & & 0,9 & 0,964 & 0,716 & 4,81 & \\
\hline $600-T_{2}$ & 315,2 & 0,97 & 3 & 0,8 & 8,26 & 3,48 & 1,92 & 7,74 & 7,74 & 1,01 & 0,88 & 0,994 & 0,747 & 2,87 & 0,21 \\
\hline $600-\mathrm{AF}$ & 380 & 1,17 & 0,52 & & 3,7 & 2,61 & 2,35 & 4,33 & 4,33 & 2,61 & $\sim 0$ & $\sim 1$ & $\sim 1$ & 2,47 & \\
\hline $900-P$ & 258,9 & 0,80 & 18,5 & & 9,8 & 5,12 & 3,47 & & & & 0,52 & 0,861 & 0,763 & 30,9 & \\
\hline $900-\mathrm{T}_{1}$ & 290 & 0,89 & 9 & & 10,0 & 4,82 & 3,11 & 2,66 & & & 0,61 & 0,952 & 0,812 & 14,5 & \\
\hline $900-\mathrm{T}_{2}$ & 316,2 & 0,97 & 5,2 & 1 & 8,34 & 4,54 & 2,96 & 24,4 & 24,4 & 3,35 & 0,59 & 0,999 & 0,861 & 6,56 & 2,72 \\
\hline $900-\mathrm{AF}$ & 380 & 1,17 & 0,52 & & 5,53 & 3,91 & 3,52 & 5,53 & 5,53 & 3,91 & $\sim 0$ & $\sim 1$ & $\sim 1$ & 4,83 & \\
\hline $1200-\mathrm{P}$ & 230,7 & 0,71 & 29 & & 12,1 & 6,97 & 5,2 & & & & 0,31 & 0,799 & 0,762 & 73,0 & \\
\hline $1200-\mathrm{T}_{1}$ & 273,6 & 0,84 & 16 & & 11,2 & 6,16 & 4,42 & 3,53 & & & 0,43 & 0,923 & 0,846 & 36,8 & \\
\hline $1200-\mathrm{T}_{2}$ & 310,6 & 0,95 & 6,8 & 1,2 & 9,51 & 5,65 & 4,13 & 34,4 & 34,4 & 4,84 & 0,41 & 0,997 & 0,922 & 12,2 & 6,66 \\
\hline $1200-\mathrm{AF}$ & 380 & 1,17 & 0,52 & & 7,38 & 5,22 & 4,7 & 7,38 & 7,38 & 5,22 & $\sim 0$ & $\sim 1$ & $\sim 1$ & 4,84 & \\
\hline
\end{tabular}

$\mathrm{P}=$ filtro passivo; $\mathrm{T}_{1}=\mathrm{BF}-$ boost $\mathrm{T}_{2}=\mathrm{BF}$ - boost modificado, $\mathrm{AF}-$ boost .

$\mathrm{P}_{\mathrm{o}}$ : potência de saída; $\mathrm{U}_{\mathrm{o}}$ : tensão de saída; $\mathrm{U}_{\mathrm{Sp}}$ : valor de pico da tensão de entrada; $\mathrm{I}_{\mathrm{g}}$ : corrente (eficaz e pico) de entrada; $\mathrm{I}_{\mathrm{S}}$ : corrente no interruptor (eficaz e pico); $\mathrm{I}_{\mathrm{La}}$ : corrente no indutor auxiliar (eficaz e pico);

TABELA II. COMPARAÇÃO ENTRE RETIFICADORES PARA DIFERENTES POTÊNCIAS: VOLUME E PERDAS

\begin{tabular}{|c|c|c|c|c|c|c|c|c|c|c|c|}
\hline $\begin{array}{c}\mathrm{P}_{\mathrm{o}} \\
{[\mathrm{W}]}\end{array}$ & $\begin{array}{c}\mathrm{V}_{\mathrm{ext}} \\
{\left[\mathrm{cm}^{3}\right]}\end{array}$ & $\begin{array}{c}\mathrm{V}_{\mathrm{fe}} \\
{\left[\mathrm{cm}^{3}\right]}\end{array}$ & $\begin{array}{c}\mathrm{V}_{\text {ext }} \\
\mathrm{aux} \\
{\left[\mathrm{cm}^{3}\right]}\end{array}$ & $\begin{array}{c}\mathrm{V}_{\mathrm{fe}} \\
\mathrm{aux} \\
{\left[\mathrm{cm}^{3}\right]}\end{array}$ & $\begin{array}{c}\mathrm{P}_{\mathrm{Cu}}{ }^{*} \\
{[\mathrm{~W}]}\end{array}$ & $\begin{array}{c}\mathrm{P}_{\mathrm{Fe}}^{*} \\
{[\mathrm{~W}]}\end{array}$ & $\begin{array}{c}\mathrm{P}_{\text {rect }} \\
{[\mathrm{W}]}\end{array}$ & $\begin{array}{c}\mathrm{P}_{\text {switch }} \\
{[\mathrm{W}]}\end{array}$ & $\begin{array}{c}\mathrm{P}_{\text {cap }} \\
{[\mathrm{W}]}\end{array}$ & $\begin{array}{c}\text { Área do } \\
\text { dissipador } \\
{\left[\mathrm{cm}^{2}\right]}\end{array}$ & $\begin{array}{c}\text { Volume } \\
\text { do filtro } \\
\text { de IEM } \\
{\left[\mathrm{cm}^{3}\right]}\end{array}$ \\
\hline $600-\mathrm{P}$ & 48 & 38,4 & & & 4,08 & 0,91 & 4,08 & & & & \\
\hline $600-\mathrm{T}_{1}$ & 38,4 & 30,7 & & & 3,22 & 0,73 & 3,96 & 1,98 & & 11 & \\
\hline $600-\mathrm{T}_{2}$ & 26,1 & 21,5 & 3,58 & 2,82 & 2,59 & 0,58 & 3,84 & 1,94 & 0,92 & 11 & \\
\hline $600-\mathrm{AF}$ & 6,84 & & & & 5,3 & 1,47 & 4,70 & 20,5 & & 149 & 131 \\
\hline $900-\mathrm{P}$ & 141 & 112 & & & 13,6 & 2,67 & 6,94 & & & & \\
\hline $900-\mathrm{T}_{1}$ & 90 & 72 & & & 7,04 & 1,71 & 6,11 & 3,14 & & 17,6 & \\
\hline $900-\mathrm{T}_{2}$ & 48 & 38,4 & 26,1 & 21,5 & 6,01 & 1,42 & 5,92 & 3,18 & 7,76 & 17,9 & \\
\hline $900-\mathrm{AF}$ & 10,5 & & & & 3,35 & 2,84 & 7,04 & 31,8 & & 282 & 194 \\
\hline $1200-\mathrm{P}$ & 262 & 206 & & & 25,8 & 4,88 & 10,4 & & & & \\
\hline $1200-\mathrm{T}_{1}$ & 164 & 131 & & & 14,8 & 3,11 & 8,84 & 4,5 & & 25,6 & \\
\hline $1200-\mathrm{T}_{2}$ & 75 & 60 & 48 & 38,4 & 10,5 & 2,33 & 8,26 & 4,64 & 15,2 & 26,3 & \\
\hline $1200-\mathrm{AF}$ & 21,3 & & & & 3,76 & 2,81 & 9,40 & 43,6 & & 495 & 194 \\
\hline
\end{tabular}

* Inclui o indutor auxiliar para $\mathrm{T} 2$

** Inclui o transistor e os diodos

$\mathrm{P}_{\mathrm{o}}$ : potência de saída; $\mathrm{V}_{\text {ext }}$ : volume externo do indutor; $\mathrm{V}_{\mathrm{fe}}$ : volume do núcleo; $\mathrm{P}$ : perda de potência no enrolamento $\left(\mathrm{P}_{\mathrm{Cu}}\right)$, no núcleo $\left(\mathrm{P}_{\mathrm{Fe}}\right)$, no retificador de entrada $\left(\mathrm{P}_{\mathrm{rect}}\right)$, no interruptor e no diodo auxiliar $\left(\mathrm{P}_{\text {switch }}\right)$ e no capacitor auxiliar $\left(\mathrm{P}_{\text {cap }}\right)$.

\section{F. Perdas}

As perdas de potência associadas aos elementos magnéticos e ao retificador de entrada foram calculadas para todas as topologias e níveis de potência.

Foi suposta uma queda de $1 \mathrm{~V}$ por diodo e, sendo conhecida a corrente média de entrada, determina-se a potência. No retificador de entrada não há perda de comutação significativa em nenhuma das topologias.

Para os indutores foram consideradas as perdas no núcleo (com base em dados de catálogo que indicam as perdas em função da freqüência, do valor de B e da massa do núcleo) e no enrolamento.

Os valores não são significantemente diferentes entre si, exceto pelas perdas nos enrolamentos, que são muito maiores nos indutores das topologias com comutação em baixa frequiência.

A razão para tal é que foi utilizado, para efeito comparativo, o mesmo valor de densidade de corrente (5 $\mathrm{A} / \mathrm{mm}^{2}$ ) no dimensionamento de todos indutores. Este não é um bom valor para indutores de baixa freqüência, uma vez que o número de espiras exigido implica em uma resistência elevada.

As perdas no capacitor auxiliar, associadas à sua resistência série equivalente, (topologia T2) têm valor próximo às do indutor, por causa do alto valor da corrente eficaz que circula por $\mathrm{C}_{\text {aux }}$.

Para os semicondutores (exceto a ponte retificadora) foram utilizados dois métodos diversos. Para os circuitos de baixa frequiência as perdas foram estimadas considerando tensão e corrente médias pelo transistor e diodo, desprezando perdas de comutação.

Para a topologia AF - boost, foi tomado como base um valor de rendimento global de $95 \%$, freqüentemente reportado na literatura, válido para a faixa de potência estudada. Subtraindo as outras perdas estimadas anteriormente, atribui-se a diferença ao transistor e ao diodo. Os valores obtidos são cerca de dez vezes maiores do que aqueles dos circuitos com comutação em baixa frequiência, 
podendo-se atribuir este aumento essencialmente às perdas de comutação.

\section{G. Dissipador de calor}

Para todos os conversores foi suposto que o os interruptores (diodo e transistor) tivessem encapsulamento TO-247 (resistência térmica $\mathrm{R}_{\theta \mathrm{jc}}=0,7^{\circ} \mathrm{C} / \mathrm{W}$ ).

A área do dissipador de calor foi calculada considerando a resistência térmica de uma placa de alumínio brilhante de 1 mm de espessura, em posição vertical [14].

Os valores elevados obtidos para a topologia com comutação em alta freqüência têm um importante impacto no volume total destes conversores.

\section{H. Filtro de Interferência Eletromagnética - IEM}

Devido à comutação em baixa freqüência, as topologias com filtro passivo, T1 e T2 não necessitam de filtros de IEM, o que já não ocorre com o AF - boost.

Uma vez que o projeto de tais filtros não é objeto deste artigo, a Tabela II indica o volume de filtros comerciais (atenuação entre 50 e $60 \mathrm{~dB} \mu \mathrm{V}$ entre $150 \mathrm{kHz}$ e $30 \mathrm{MHz}$ ). Note-se que o volume do filtro é muito maior do que o do indutor, sendo similar ao volume do indutor estipulado para o retificador com filtro passivo.

\section{Circuitos eletrônicos}

$\mathrm{O}$ circuito eletrônico usado tanto em T1 quanto em T2 é essencialmente um gerador de pulso sincronizado com a rede. Se for implementada a regulação da tensão de saída, torna-se necessário um circuito adicional para ajustar a largura do pulso. Este circuito poderia ser facilmente implementado, por exemplo, com um TCA 785.

Para o retificador com comutação em alta freqüência, seria empregado um circuito integrado comercial, que exige três sinais de entrada: tensão retificada, tensão de saída e corrente pelo indutor.

Embora este último caso apresente uma complexidade um pouco maior, não há diferenças muito importantes neste aspecto entre os retificadores.

Obviamente, o retificador com filtro passivo não faz uso de nenhum circuito eletrônico.

\section{RESULTADOS EXPERIMENTAIS}

As figuras 6 e 7 mostram formas de onda para as topologias T1 e T2. Em ambos os casos o conteúdo harmônico está dentro dos limites da norma.

$\mathrm{O}$ valor de indutância utilizado para T1 é aquele indicado na Tabela I. Os resultados experimentais são consistentes com os teóricos apresentados na mesma tabela.

Para a topologia T2, por ter sido testada em $60 \mathrm{~Hz}, \mathrm{o}$ valor foi recalculado, obtendo-se, como esperado, valores inferiores àqueles de $50 \mathrm{~Hz}$. O esforço de corrente é de $20 \mathrm{~A}$. $\mathrm{O}$ fator de potência medido foi 0,85 , sendo determinado pela distorção da corrente, que equivale a uma DHT de $59 \%$.

$\mathrm{O}$ rendimentos da topologia $\mathrm{T} 1$ foi de $97 \%$, enquanto para T2 foi obtido $96 \%$, o que é coerente com os valores expressos na Tabela II. O menor rendimento de T2 deve-se ao aumento das perdas devido ao modo de funcionamento do circuito auxiliar.

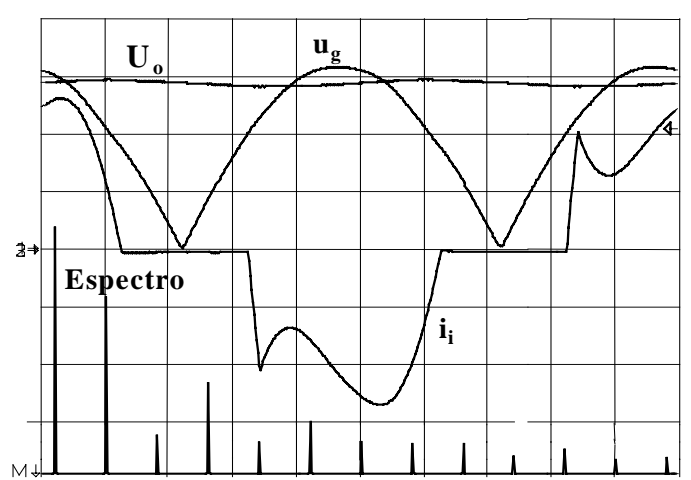

Fig. 6 - Tensão de entrada retificada (100 V/div), corrente de entrada ( $2 \mathrm{~A} / \mathrm{div})$ e seu espectro $\left(0,4 \mathrm{~A}_{\mathrm{rms}} / \mathrm{div}\right)$ $\mathrm{U}_{\mathrm{i}}=225 \mathrm{~V}_{\mathrm{rms}}, \mathrm{U}_{\mathrm{o}}=284 \mathrm{~V}, 50 \mathrm{~Hz}$ e $\mathrm{P}_{\mathrm{o}}=600 \mathrm{~W}$.

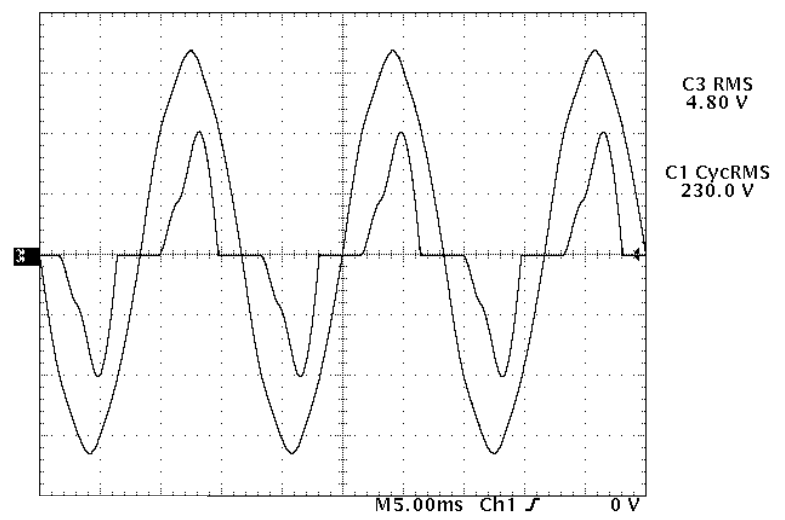

Fig. 7 - Tensão de entrada $U_{\mathrm{i}}(100 \mathrm{~V} /$ div $)$, corrente de entrada $i_{\mathrm{i}}(5$ A/div) $U_{i}=230 \mathrm{~V}_{\mathrm{rms}}, \mathrm{U}_{\mathrm{o}}=292 \mathrm{~V}, \mathrm{P}_{\mathrm{o}}=900 \mathrm{~W}, 60 \mathrm{~Hz}, \mathrm{~L}=6 \mathrm{mH}$, $\mathrm{L}_{\mathrm{a}}=1 \mathrm{mH}, \mathrm{C}_{\mathrm{a}}=44 \mu \mathrm{F}$.

\section{CONCLUSÕES}

Esta análise comparativa entre retificadores de alta qualidade empregando comutação em alta ou em baixa freqüência visou dar informações para a escolha de uma solução que considere aspectos relacionados com o volume dos elementos magnéticos empregados, o volume total do retificador, a possibilidade de regulação da tensão de saída, a geração de IEM e outros aspectos, sempre garantindo a conformidade com normas de distorção da corrente.

As topologias que empregam comutação em baixa freqüência têm como principal vantagem a não necessidade de uso de filtro de IEM e a eliminação das perdas de comutação, o que implica numa drástica diminuição do volume dos dissipadores, resultando ainda num maior rendimento.

Para valores de potência mais elevados (acima de 900 $\mathrm{W}$ ), os tamanhos do dissipador e do filtro de IEM se tornam muito significativos no conversor com comutação em alta freqüência, enquanto o indutor se torna muito grande na solução passiva.

Por outro lado, pode-se esperar uma redução do volume total do conversor com o emprego das topologias T1 e, principalmente, $\mathrm{T} 2$.

A topologia T2, mesmo utilizando dois indutores, 
minimiza o volume do conversor. Sua principal desvantagem é o esforço de corrente presente nos componentes do circuito auxiliar, o que resulta num menor rendimento em comparação com T1.

Em termos das perdas totais, a solução de alta frequiência apresenta os piores resultados devido às perdas de comutação dos interruptores. A grande vantagem do AF - boost é a regulação da tensão de saída, que permite compensar plenamente variações na tensão de entrada e na carga. Já as outras topologias (exceto a passiva) conseguem compensar reduções da corrente de carga até cerca de 50\% do valor nominal (valores obtidos experimentalmente), enquanto praticamente não têm atuação frente a variações na tensão de entrada.

Caso a aplicação não exija este tipo de regulação (por exemplo, se os conversores alimentados pela saída CC puderem operar com variação nesta tensão) e aspectos de densidade volumétrica de potência e simplicidade de operação do conversor forem mais relevantes, as topologias T1 ou T2 podem ser uma escolha conveniente.

\section{AGRADECIMENTOS}

Os autores desejam agradecer à Fundação de Amparo à Pesquisa do Estado de São Paulo (FAPESP) pelos financiamentos das visitas do Dr. Spiazzi e do Dr. Buso ao Brasil e do projeto 99/09400-2.

\section{REFERÊNCIAS BIBLIOGRÁFICAS \\ [1] IEC 61000-3-2, International Electrotechnical} Commission, 3, Genève, Switzerland, 1998.

[2] M. Jovanovic, D. E. Crow, "Merits and Limitations of Full-Bridge Rectifier with LC Filter in Meeting IEC 1000-3-2 Harmonic-Limit Specifications," Proc. of the IEEE Applied Power Electronics Conf.. (APEC), pp. 354360, March 1996.

[3] I. Suga, M. Kimata, Y. Ohnishi, R. Uchida. "New Switching Method for Single-Phase AC to DC Converter," Proc. of the Power Conversion Conference (PCC), pp.93-98, 1993.

[4] L. Rossetto, G.Spiazzi, P. Tenti, "Boost PFC with $100 \mathrm{~Hz}$ Switching Frequency Providing Output Voltage Stabilization and Compliance with EMC Standards", Proc. of the Industry Applications Society Annual Meeting, St. Louis, USA, pp. 1567-1573, 1998.

[5] G. Spiazzi, S. Buso, "A Line-Frequency Commutated Rectifier Complying with IEC 1000-3-2 Standards," Proc. of the IEEE Applied Power Electronics Conf., March 1999.

[6] J. A. Pomilio, G. Spiazzi, "A Double-Line-Frequency Commutated Rectifier Complying with IEC 1000-3-2 Standards" Proc. of the IEEE Applied Power Electronics Conf., March 1999.

[7] J. A. Pomilio, G. Spiazzi, "A Low-Inductance LineFrequency Commutated Rectifier Complying with IEC 1000-3-2 Standards," CD record. of the IEEE Power Electronics Specialists Conf. (PESC), June 1999.

[8] Y. Shimma and K. Iida: "Inverter applications to air conditioning field", Proc. Of IPEC 2000, May 2000, pp. 1747-1750.
[9] J. Salmon and D. Koval: "Improving the operation of 3phase diode rectifiers using an asymmetrical half-bridge dc-link active filter", CD record of IAS Annual Meeting, October 2000.

[10] E. L. M. Mehl and I. Barbi: "An improved high-power factor and low-cost three-phase rectifier", IEEE Trans. on Industry Applications, vol. 33, no. 2, pp. 485-492, March-April 1997.

[11] S. Hansen, P. N. Enjeti, J. Han and F. Blaabjerg: "An integrated single-switch approach to improve harmonic performance af standard PWM adjustable speed drives", Proc. of IAS Annual Meeting, pp. 789-795, Oct. 1999.

[12] B. Mammano, L. Dixon: "Choose the Optimum Topology for High Power Factor Supplies", PCIM Magazine, pp. 8-18, March 1991.

[13] EN 61000-3-2 prA14, European Committees for Electrotechnical Standardization - CENELEC, Brussels, Belgium, March 2000.

[14] D. S. Steinberg: "Cooling Techniques for Electronic Equipment”, John Wiley \& Sons, Inc., 1980.

\section{DADOS BIOGRÁFICOS}

José Antenor Pomilio nasceu em Jundiaí - SP em 1960. É engenheiro eletricista (1983), Mestre (1986) e Doutor em Eng. Elétrica (1991) pela Universidade Estadual de Campinas. De 1988 a 1991 foi chefe do grupo de eletrônica de potência do Laboratório Nacional de Luz Síncrotron. Em 1993/1994 realizou um estágio de pós-doutoramento junto à Universidade de Pádua - Itália. Foi editor da revista Eletrônica de Potência (1999/2000). Atualmente é presidente da Sociedade Brasileira de Eletrônica de Potência e membro eleito do Comitê Administrativo da The IEEE Power Electronics Society. É professor da Faculdade de Engenharia Elétrica e de Computação da Unicamp deste 1984. Suas principais áreas de interesse são técnicas de eletrônica de potência aplicadas à qualidade da energia elétrica, condicionamento elétrico aplicado a fontes alternativas de energia.

Giorgio Spiazzi nasceu em Legnago (província de Verona, Itália) em 1962. Graduou-se em Engenharia Elétrica na Universidade de Padova em 1988. Em 1993 obteve seu doutorado em Eletrônica Industrial e Informática no Departamento de Eletrônica e Informática da mesma Universidade, onde é professor desde 1993. Suas áreas de interesse são técnicas de controle avançadas para conversores de potência, pré-conversores de alto fator de potência e técnicas de comutação suave.

Simone Buso nasceu em Pádua, Itália em 1968. Recebeu os graus de mestre em Engenharia Elétrica e de Doutor em Eletrônica Industrial pela Universidade de Pádua, em 1992 e 1997, respectivamente. Desde 1993 é pesquisador junto ao Departamento de Eletrônica e Informática da mesma universidade. Seus principais interesses de pesquisa incluem conversores $\mathrm{CC} / \mathrm{CC}$, circuitos integrados "smart power", controle digital e controle robusto de conversores de potência. 